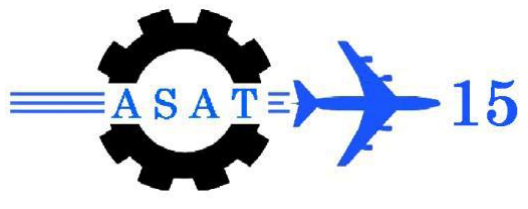

\title{
An Analytical Model for Repulsive Electrostatic Microactuator Using a Conformal Mapping Technique
}

\author{
M. B. Tayel ${ }^{*}$, A. M. El Masry ${ }^{\dagger}$
}

\begin{abstract}
Electrostatic microactuators are able to produce a repulsive force in the out-ofplane direction. The electrostatic microactuators use an asymmetric electric field surrounding the top and bottom surfaces of the moving fingers to produce a repulsive force. The displacement of moving finger is not limited by the "pull-in" effect. In addition, the usage of a repulsive force leads to the elimination of the sticking problem. In this paper, an analytical model for the repulsive-force, and the fringe capacitance of a microactuator is developed putting the moving plate thickness in consideration using conformal mapping techniques. With this technique, electric field lines are geometrically approximated to separately model the different capacitive components. These components are finally combined to obtain the equivalent fringe capacitance. The model reveals the maximum out-of-plane displacement. Rules are derived based on the analytical model for tuning and optimization of the microactuator performance. Simulations are conducted to verify the analytical model. In addition, the mechanism for generating the repulsive force is explained.
\end{abstract}

\section{Introduction}

Micro-Electro-Mechanical Systems (MEMS) are integrated micro devices or systems combining electrical, electronic, and mechanical components. Trend toward smaller size, higher performance, and greater functionality for electronic devices is possibly made by the success of solid-state microelectronics technology [1]. An early application of MEMS was in the field of microsensor and microactuator for measuring or driving position, pressure, velocity, acceleration, force, torque, flow, magnetic field, temperature, gas composition, humidity, $\mathrm{pH}$, fluid ionic concentration, and biological gas/liquid/Molecular concentration [2].There are four major actuation technologies used in Micro-Electro-Mechanical System (MEMS) devices, namely electrostatic actuation, electromagnetic actuation, thermal actuation, and piezoelectric actuation. Electrostatic actuation is the most commonly used due to its high compatibility with IC fabrication technology, low power consumption, quick response, and simple structure [3]. A variety of electrostatic microactuators employing arrangements such as parallel plate capacitors, interdigitated finger or comb structures and rotor/stator designs have been fabricated [4]. There are two types of forces used in electrostatic (ES) microactuators: attractive force and repulsive force.

Some applications have been successfully commercialized in market such as thermal inkjet printer, automotive accelerometer, Digital light projection (DLP) systems, modulators, disposable bio-analysis chip and airbag crash sensor [2]. MEMS is also widely used to fabricate micro optical components or optical systems such as deformable micromirror array

Faculty of Engineering, Alexandria University, Egypt, profbasyouni@gmail.com

Faculty of Engineering, Alexandria University, Egypt, amasrawy@yahoo.com 
for adaptive optics, optical scanner for bar code scanning, optical switching for fiber optical communication etc. This special field of MEMS is called 'Micro-Opto-Electro-Mechanical Systems (MOEMS)'.

Any conformal function preserves potential and flux functions and capacitances between corresponding conductors. This property is the key for using conformal mapping in capacitance calculation [5,6]. Most capacitance calculations for parallel-plate condensers assume that the plates are infinite planes and neglect the fringing effect [7]. This assumption is equivalent to saying that only a uniform homogeneous field region between the two plates. However, the resulting fringing field has a strong effect on the value of capacitance and on the ES force. Therefore, it must be taken into consideration when one wants to obtain a precise value of the capacitance and its effecting on ES force.

\section{Repulsive Microactuator Design}

The repulsive microactuator has a two-layer finger structure, as shown in Figure (1). All fixed fingers are attached to the substrate, whereas all moving fingers are connected together and are supported by suspension springs, not shown in Figure (1). Figure (2) is a cross section of the two-layer finger structure of this microactuator.

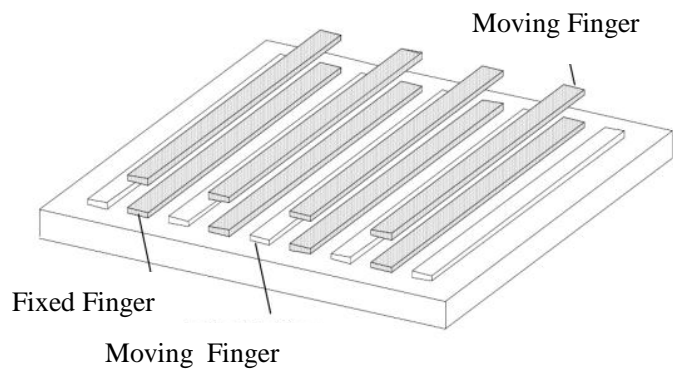

Figure (1): 3-D view of repulsive microactuator fingers

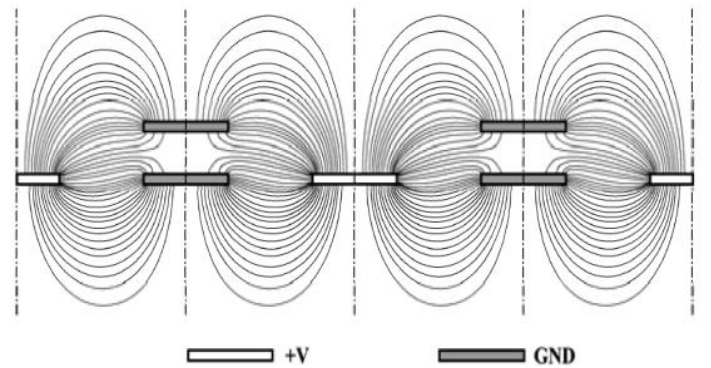

Figure (2): Cross section of the microactuator

According to the virtual work theory, the force exerted on moving fingers of the microactuator, positive in the upward direction can be expressed as

$$
\mathrm{F}=\frac{\mathrm{dE}}{\mathrm{dH}}
$$

where $\mathrm{H}$ is the distance between the fixed and moving fingers.

$$
\mathrm{E}=\frac{1}{2} \mathrm{~V}^{2} \mathrm{C}
$$

$\mathrm{E}$ is the energy stored in the finger structure, $\mathrm{V}$ is the applied voltage and 


$$
\mathrm{C}=\mathrm{L} . \mathrm{C}_{\mathrm{unit}}
$$

is the total capacitance between the moving fingers and fixed fingers, $\mathrm{C}_{\mathrm{unit}}$ is the capacitance per unit length (L) and $\mathrm{C}_{\text {cell }}$ is the capacitance in each elementary cell and $\mathrm{N}$ is the number of moving fingers, figure (3-a),

$$
\mathrm{C}_{\text {unit }}=2 \mathrm{~N} . \mathrm{C}_{\text {cell }}
$$

Substituting equations (2), (3) and (4) into equation (1) to get

$$
\mathrm{F}=\frac{\mathrm{V}^{2} \cdot \mathrm{L}}{2} \cdot \frac{\mathrm{dC}_{\mathrm{unit}}}{\mathrm{dH}}
$$

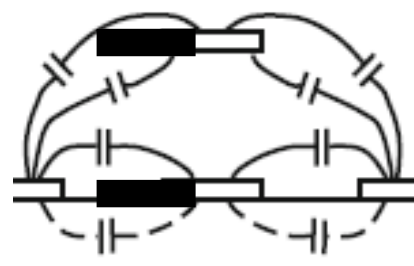

(a)

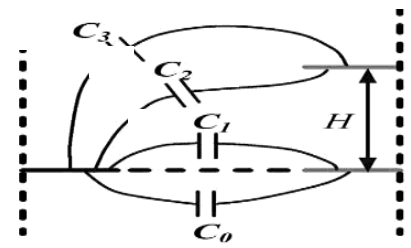

(b)

Figure (3) (a) A unit cell (b) An elementary cell.

Figure (3-b) shows an elementary cell with the line connecting the fixed fingers (lateral capacitance) and the line connecting the fixed and moving fingers (fringing capacitance) which can be approximated by a Neumann boundary line. The elementary cell capacitances can be divided into two parts, the fringe and lateral parts, as shown in Figure (4). The two parts are denoted as the fringe capacitance Ccell_fringe and the lateral capacitance Ccell_lateral, i.e.

$$
\mathrm{C}_{\text {cell }}=\mathrm{C}_{\text {cell_fringe }}+\mathrm{C}_{\text {cell_lateral }}
$$

where

$\mathrm{C}_{\text {cell_fringe }}=\mathrm{C}_{\text {corner }}+\left(\mathrm{C}_{\mathrm{sw}, \text { top1 } 1}+\mathrm{C}_{\mathrm{sw}, \text { top2 }}\right)+\left(\mathrm{C}_{\text {top,top1 } 1}+\mathrm{C}_{\text {top,top2 }}\right)+\mathrm{C}_{\mathrm{sw} 1, \mathrm{sw} 2}$

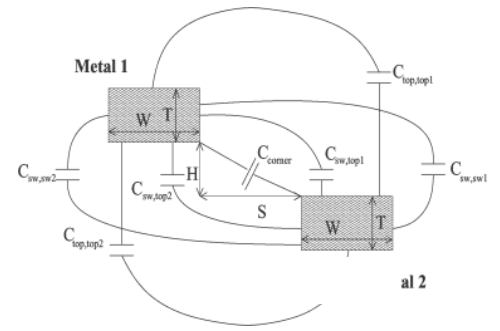

(a) Fringe capacitance

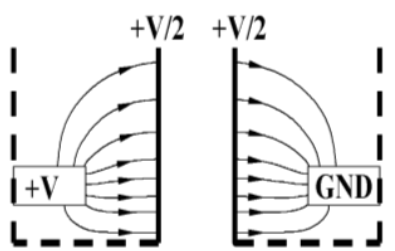

(b) Lateral capacitance

Figure (4) The elementary cell capacitances

Substituting equations (4) and (6) into equation (5) to get

$$
\mathrm{F}=\mathrm{F}_{\mathrm{o}}\left(\frac{\mathrm{d}}{\mathrm{dh}} \mathrm{C}_{\text {cell_fringe }}+\frac{\mathrm{d}}{\mathrm{dh}} \mathrm{C}_{\text {cell_lateral }}\right)
$$

where $F_{o}=\frac{N \cdot V^{2} \cdot L}{W}$ is the force constant. The finger width $\mathrm{w}$ is selected to normalize the dimensions of the cross section of the microactuator, and $\mathrm{h}=\mathrm{H} / \mathrm{w}$. 


\section{Capacitance Analytical Model}

Figure (4-a) shows the EF lines between the two fixed plates at the same layer. Figure (4-b) shows the EF lines between the sidewall of the top plate (moving plate) and the top of the bottom plate (fixed plate) that contribute to the capacitances $\mathrm{C}_{\mathrm{sw} \text {,top1 }}$ and $\mathrm{C}_{\text {corner }}$ [8]. Note that the EF lines between the sidewall of the bottom plate and the bottom surface of the top plate, which contribute to $\mathrm{C}_{\mathrm{sw}, \mathrm{top} 2}$, can be shown in a similar way.

\subsection{The Sidewall-Top Capacitance $\left(\mathrm{C}_{\mathrm{sw}, \text { top }}\right)$}

To calculate the fringe capacitance components, map these perpendicular surfaces to an equivalent parallel-plate system using conformal mapping [9]. This can be done if and only if the elliptical system is confocal.

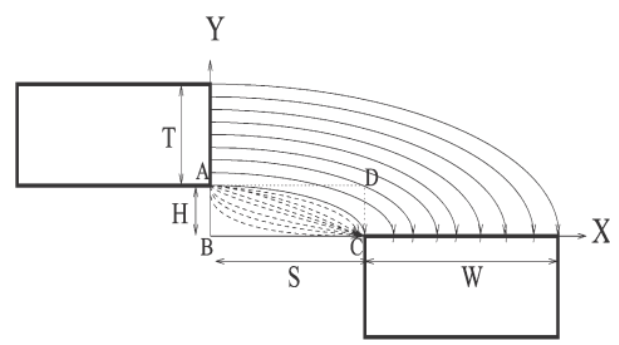

Figure (5): Electric field lines between the two non overlapping plates.

Figure (5) shows the EF lines between the two non overlapping plates contributing to $\mathrm{C}_{\mathrm{sw} \text {,top }}$. Dashed lines show the corners fringing EF components. The derived expression for the sidewall-top capacitance $\mathrm{C}_{\mathrm{sw} \text {,top }}$ is

$$
\mathrm{C}_{\mathrm{sw}, \mathrm{top}}=\frac{\varepsilon \mathrm{di}}{\pi / 2} \ln \frac{\frac{\mathrm{h}}{\mathrm{s}}+\left(\frac{\mathrm{t}^{\prime}}{\mathrm{s}}\right)+\sqrt{1+\left(\frac{\mathrm{t}^{\prime}}{\mathrm{s}}\right)^{2}+2\left(\frac{\mathrm{h}}{\mathrm{s}}\right)\left(\frac{\mathrm{t}^{\prime}}{\mathrm{s}}\right)}}{1+\left(\frac{\mathrm{h}}{\mathrm{s}}\right)}
$$

\subsection{The Top Two Capacitance}

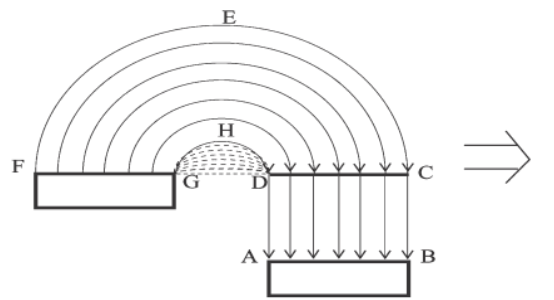

(a)

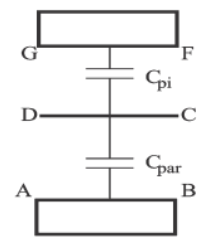

(b)

Figure (6) (a) Electric field lines contributing to $C_{p i}$ and $C_{p a r}$.

(b) Circuit model showing $\mathrm{C}_{\mathrm{pi}}$ and $\mathrm{C}_{\text {par }}$ in series.

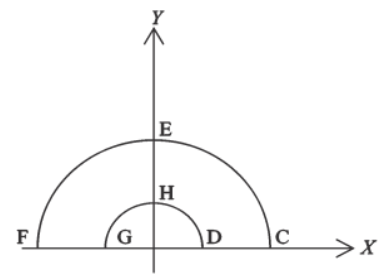

$z$ - plane

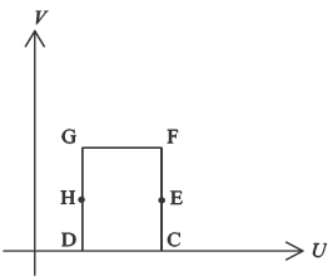

$w$ - plane

Figure (7) Radial geometry in Z-plane is mapped to linear geometry in the $W$-plane to calculate $C_{p i}$. 
Figure (6-a) shows the EF lines that contribute to $C_{\text {top,top. The equivalent capacitance model }}$ is shown in Figure (6-b).

Capacitance $\mathrm{C}_{\text {par }}$ is the parallel plate capacitance between the top surface of fixed plate (AB) and a horizontal fictitious plane [shown as line CD in Figure (6-b)] and is given by

$$
\mathrm{C}_{\text {par }}=\varepsilon_{\mathrm{di}} \frac{\mathrm{W}}{\mathrm{h}+\mathrm{t}}
$$

and Capacitance $\mathrm{C}_{\mathrm{pi}}$, due to radial field, is modeled by conformal mapping. Using the preceding transformation, the radial EF Figure (7), in the Z-plane is mapped into a linear EF in the $\mathrm{W}$-plane. In the $\mathrm{W}$-plane, the capacitance per unit length is then given by

$$
\mathrm{C}_{\mathrm{pi}}=\frac{\varepsilon_{\mathrm{di}}}{\pi} \ln \left(1+\frac{2 \mathrm{w}}{\mathrm{s}}\right)
$$

The expression for capacitance $c_{\text {top,top }}\left(\right.$ Series $\left[c_{\text {par }}, c_{\mathrm{pi}}\right]$ ) is derived as [8]

$$
c_{\text {top,top }}=\varepsilon_{\text {di }} \frac{w \ln \left(1+\frac{2 w}{s}\right)}{w \pi+(h+t) \ln \left(1+\frac{2 w}{s}\right)}
$$

The capacitance contributed by EF lines in the semicircular region GDH is approximated as

$$
\mathrm{C}_{(\mathrm{GDH})}=\frac{\varepsilon_{\mathrm{di}}}{\pi} \exp \left(\frac{-(\mathrm{t}+\mathrm{S}}{3 \mathrm{~S}}\right)
$$

To modify equation (10) using an empirical geometry-dependent factor as follows:

$$
\mathrm{C}_{\mathrm{par}}=\varepsilon_{\mathrm{di}} \frac{\mathrm{W}}{\mathrm{h}+\mathrm{t}} \exp \left(-\frac{\mathrm{h}+\mathrm{t}}{\mathrm{s}+\mathrm{W}}\right)
$$

Combining equations (12), (13), and (14), the final expression for $c_{\text {top,top }}$ can be obtained as [8]

$$
C_{\text {top,top }}=\frac{\varepsilon_{\mathrm{di}} \mathrm{W} \alpha\left(\ln \left(1+\frac{2 \mathrm{w}}{\mathrm{s}}\right)+\mathrm{e}^{\left(\frac{-(\mathrm{t}+\mathrm{S}}{3 \mathrm{~S}}\right)}\right)}{\mathrm{W} \pi \alpha+(\mathrm{h}+\mathrm{t})\left(\ln \left(1+\frac{2 \mathrm{w}}{\mathrm{s}}\right)+\mathrm{e}^{\left(\frac{-(\mathrm{t}+\mathrm{S}}{3 \mathrm{~S}}\right)}\right)}
$$

where $\alpha=\exp [-(\mathrm{h}+\mathrm{t}) /(\mathrm{s}+\mathrm{w})]$

The analytical expression for $\mathrm{C}_{\mathrm{sw}, \mathrm{sw}}$ is analogous to $\mathrm{C}_{\mathrm{top} \text {,top }}$ and can be similarly expressed as [8]

$$
\mathrm{C}_{\mathrm{sW}, \mathrm{SW}}=\frac{\varepsilon_{\mathrm{di}} \mathrm{t} \beta\left(\ln \left(1+\frac{2 \mathrm{t}}{\mathrm{h}}\right)+\mathrm{e}^{\left(-\frac{\mathrm{h}+\mathrm{W}}{3 \mathrm{~h}}\right)}\right)}{\mathrm{t} \pi \beta+(\mathrm{S}+\mathrm{W})\left(\ln \left(1+\frac{2 \mathrm{t}}{\mathrm{h}}\right)+\mathrm{e}^{\left(-\frac{\mathrm{h}+\mathrm{W}}{3 \mathrm{~h}}\right)}\right)}
$$

where $\beta=\exp [-(\mathrm{s}+\mathrm{w}) /(\mathrm{h}+\mathrm{t})]$

\subsection{Corner Capacitance Fringing Field $\mathbf{C}_{\text {corner }}$}

The fringing capacitance due to the corners $\mathrm{C}_{\text {corner }}$. is modeled based on two characteristics:

$$
\mathrm{C}_{\text {corner }}=\frac{\varepsilon_{\mathrm{di}}}{\pi} \sqrt{\frac{\mathrm{h} \mathrm{s}}{\mathrm{h}^{2}+\mathrm{s}^{2}}}
$$

By adding $\mathrm{C}_{\mathrm{sw}, \text { top }}, \mathrm{C}_{\text {corner }}, \mathrm{C}_{\text {top,top }}$, and, $\mathrm{C}_{\mathrm{sw}, \mathrm{sw}}$, the overall fringe capacitance $\mathrm{C}_{\text {cell_fringe }}$ per unit length can be obtained as 


$$
\begin{gathered}
\mathrm{C}_{\text {cell_fringe }}=4 \frac{\varepsilon_{\mathrm{di}}}{\pi} \ln \left[\frac{\mathrm{h}+\eta \mathrm{t}+\sqrt{\mathrm{S} 2+(\eta \mathrm{t}) 2+2 \eta \mathrm{ht}}}{\mathrm{s}+\mathrm{h}}\right] \\
+2 \frac{\varepsilon_{\mathrm{di}} \alpha \mathrm{W}\left(\ln \left(1+\frac{2 \mathrm{~W}}{\mathrm{~s}}\right)+\mathrm{e}^{\left(\frac{-(\mathrm{t}+\mathrm{S}}{3 \mathrm{~S}}\right)}\right)}{\pi \alpha \mathrm{W}+(\mathrm{h}+\mathrm{t})\left(\ln \left(1+\frac{2 \mathrm{~W}}{\mathrm{~S}}\right)+\mathrm{e}^{\left(\frac{-(\mathrm{t}+\mathrm{S}}{3 \mathrm{~S}}\right)}\right)} \\
+2 \frac{\varepsilon_{\mathrm{di}} \mathrm{t} \beta\left(\ln \left(1+\frac{2 \mathrm{t}}{\mathrm{h}}\right)+\mathrm{e}^{\left(-\frac{\mathrm{h}+\mathrm{W}}{3 \mathrm{~h}}\right)}\right)}{\mathrm{t} \pi \beta+(\mathrm{S}+\mathrm{W})\left(\ln \left(1+\frac{2 \mathrm{t}}{\mathrm{h}}\right)+\mathrm{e}^{\left(-\frac{\mathrm{h}+\mathrm{W}}{3 \mathrm{~h}}\right)}\right)}+\frac{\varepsilon_{\mathrm{di}}}{\pi} \sqrt{\frac{\mathrm{h} \mathrm{s}}{\mathrm{h}^{2}+\mathrm{s}^{2}}}
\end{gathered}
$$

\subsection{A Lateral Capacitance Model}

The lateral capacitance $\mathrm{C}_{\text {cell_lateral }}$ represented as $C_{A A^{\prime} B^{\prime} B}$. where $C_{A A^{\prime} B^{\prime} B}$ is the capacitance in the rectangular area AA'B'B. Three steps are used in order to achieve a conformal transformation from the $\mathrm{W}$-plane to the T-plane this is shown in Figure (8) i.e.,

a) from $\mathrm{W}$-plane to Z-plane.

b) from Z-plane to Q-plane.

c) from $Q$-plane to $T$-plane.
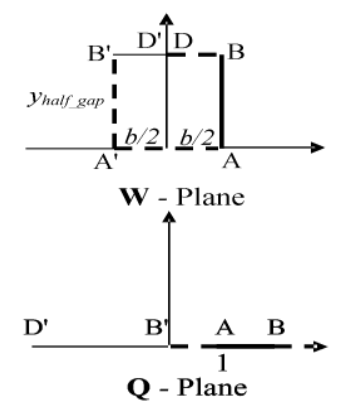

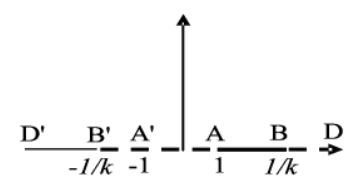

Z - Plane

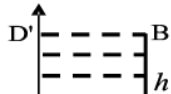

$\mathrm{B}^{\prime}=$ ニニュJA

T - Plane

Figure (8): A conformal-transformation-based method is used to derive the microcapacitance in the rectangular area $A^{A} \mathbf{A}^{\prime} B$ ' $B$.

The expression for capacitance $C_{\text {cell_lateral }}$ is derived as [4]:

$$
\mathrm{C}_{\text {cell_lateral }}=\varepsilon_{\mathrm{di}} \cdot \frac{\mathrm{j} \cdot \mathrm{sn}^{-1}\left(\infty, \mathrm{k}_{1}\right)}{\mathrm{sn}^{-1}\left(1, \mathrm{k}_{1}\right)}
$$

Substituting equation (18) and equation (19) into equation (8) to get:

$$
F=F_{o} \cdot\left(\Theta_{l}+\Theta_{f}\right)
$$

where $\Theta_{l}$ represents rate of change of capacitance $C_{\text {cell-lateral }}$ with respect to $h$ and $\Theta_{f}$ represents rate of change of capacitance $C_{\text {cell_fringe }}$ with respect to $h$.

\section{Simulations of the Introduced Model}

This section presents the simulation results for repulsive electrostatic microactuators. It shows the role of the fringe capacitance and its parameters effect, an estimate of the repulsive force generated in the microactuator and reveals the maximum out-of-plane displacement which can be achieved, fingers width and thickness effect, electrostatic vertical distance between moving and fixed fingers and lateral distance between fixed fingers effect, and applied voltage effect on the microactuator performance. 


\subsection{The Fringe Capacitance Calculation of the Introduced Model}

The fringe capacitance decreases with the increase of normalized vertical distance between the moving and the fixed fingers, figure (9).

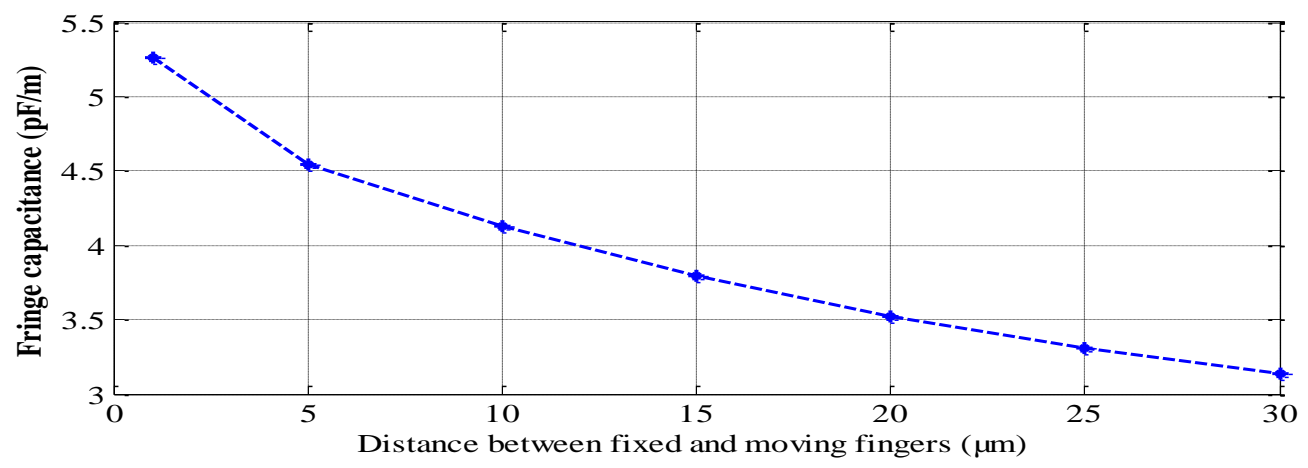

Figure (9): Calculated fringing capacitance

The effect of the fringe capacitance is dominant when the vertical distance between the fixed and the moving fingers is small in comparison to the total capacitance that shown in figure (10).

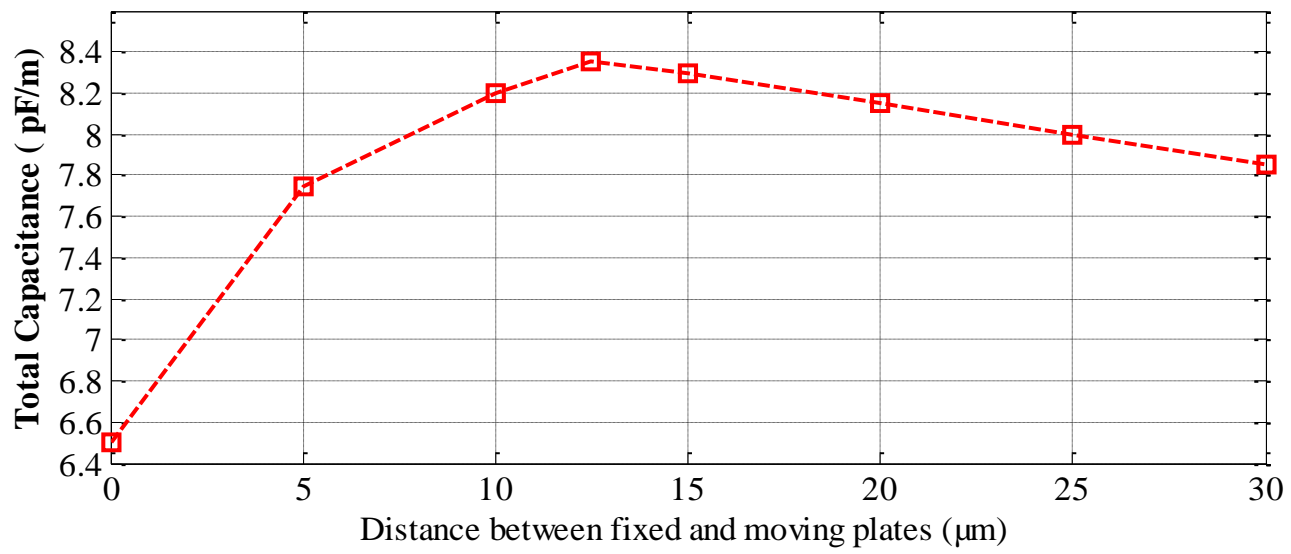

Figure (10): Total capacitance between fixed and moving plates

\subsection{The Effect of Lateral Distance Between the Fixed Plates on the Fringe Capacitance}

Decreasing the lateral distance between fixed fingers increases the fringe capacitance, figure (11). 


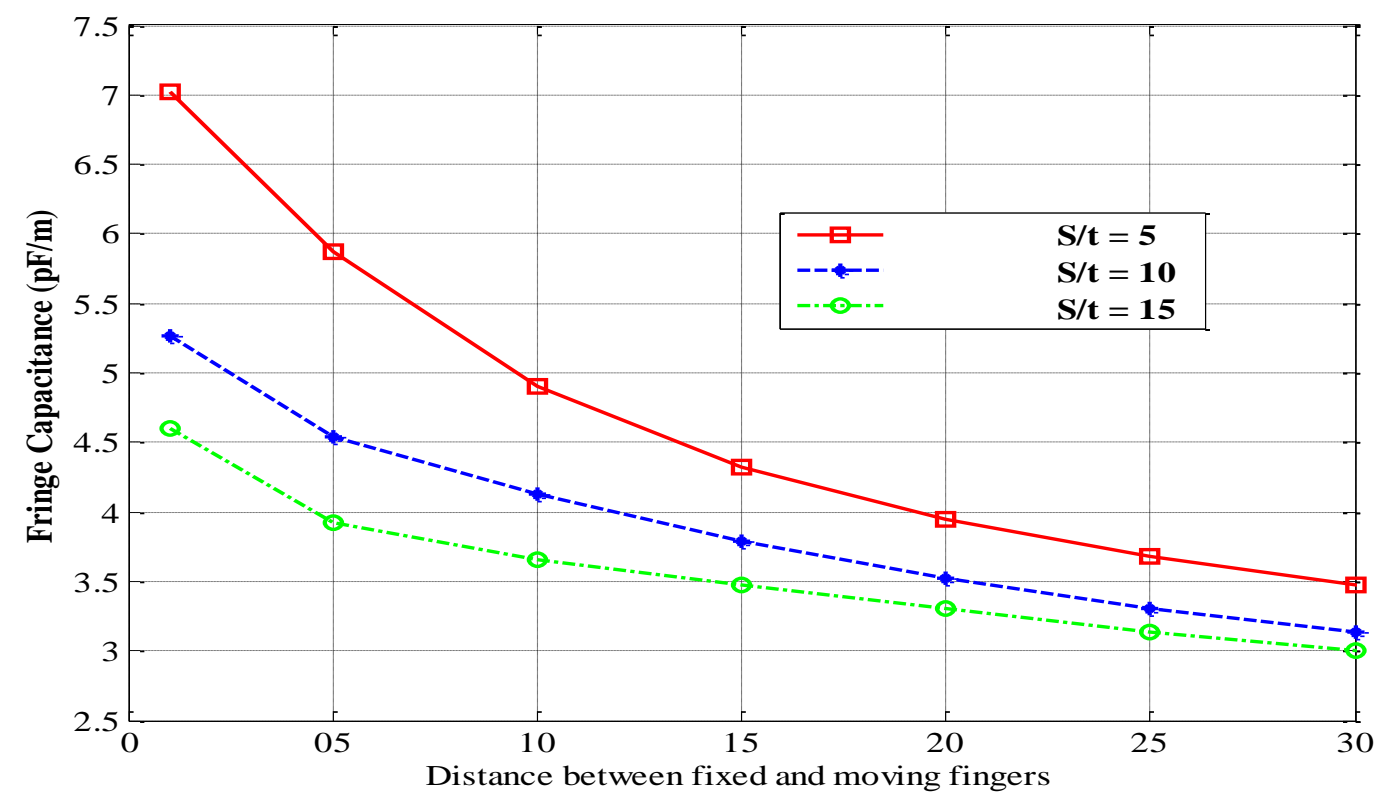

Figure (11): Effect of varying the lateral distance on the fringe capacitance

\subsection{The Effect of Finger Width on the Fringe Capacitance}

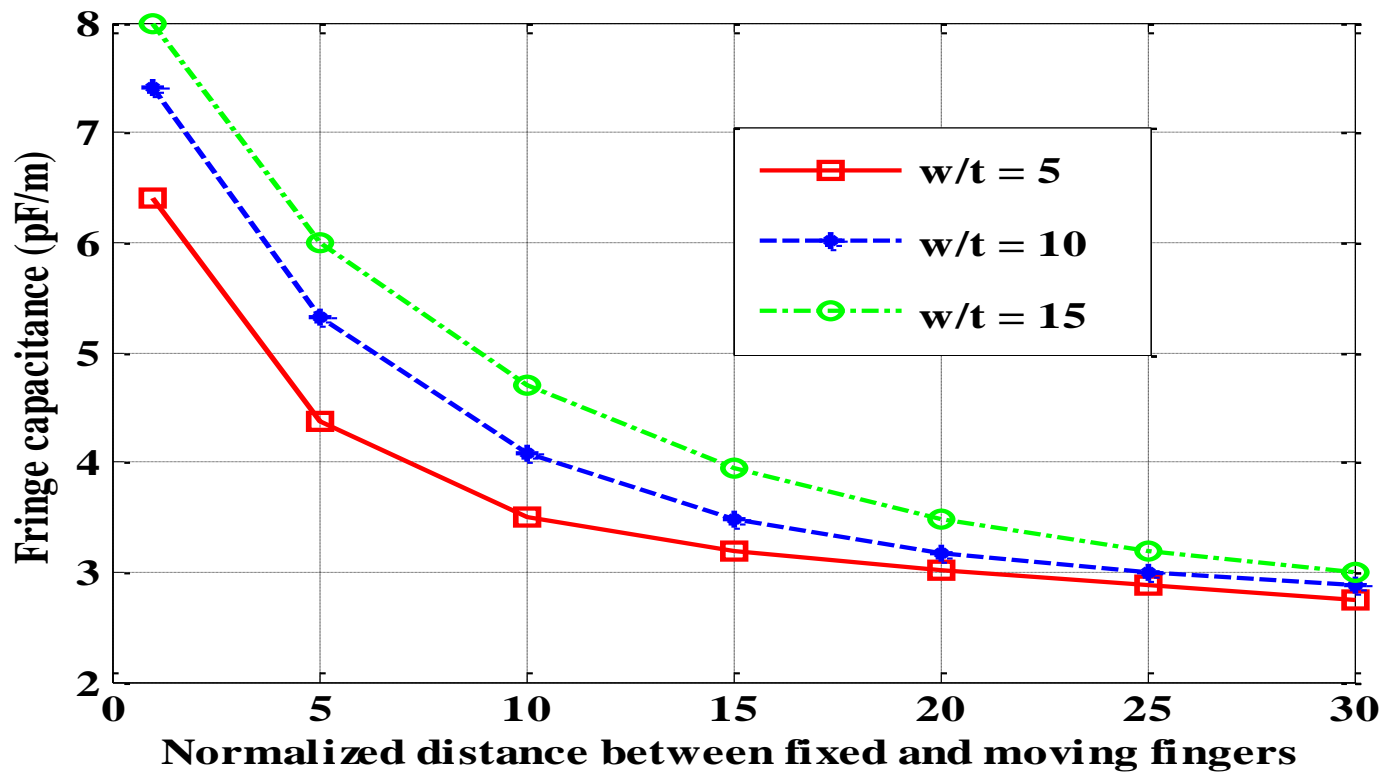

Figure (12): Effect of varying the finger width on the fringe capacitance

Increasing the width of the fixed finger increases the fringe capacitance, figure (12).

\subsection{The Effect of Repulsive Force on the Moving Finger}

Figure (13) shows that the maximum normalized vertical distance for generating a repulsive force is $h=h 0$ (where $h 0=1.35$ ). 


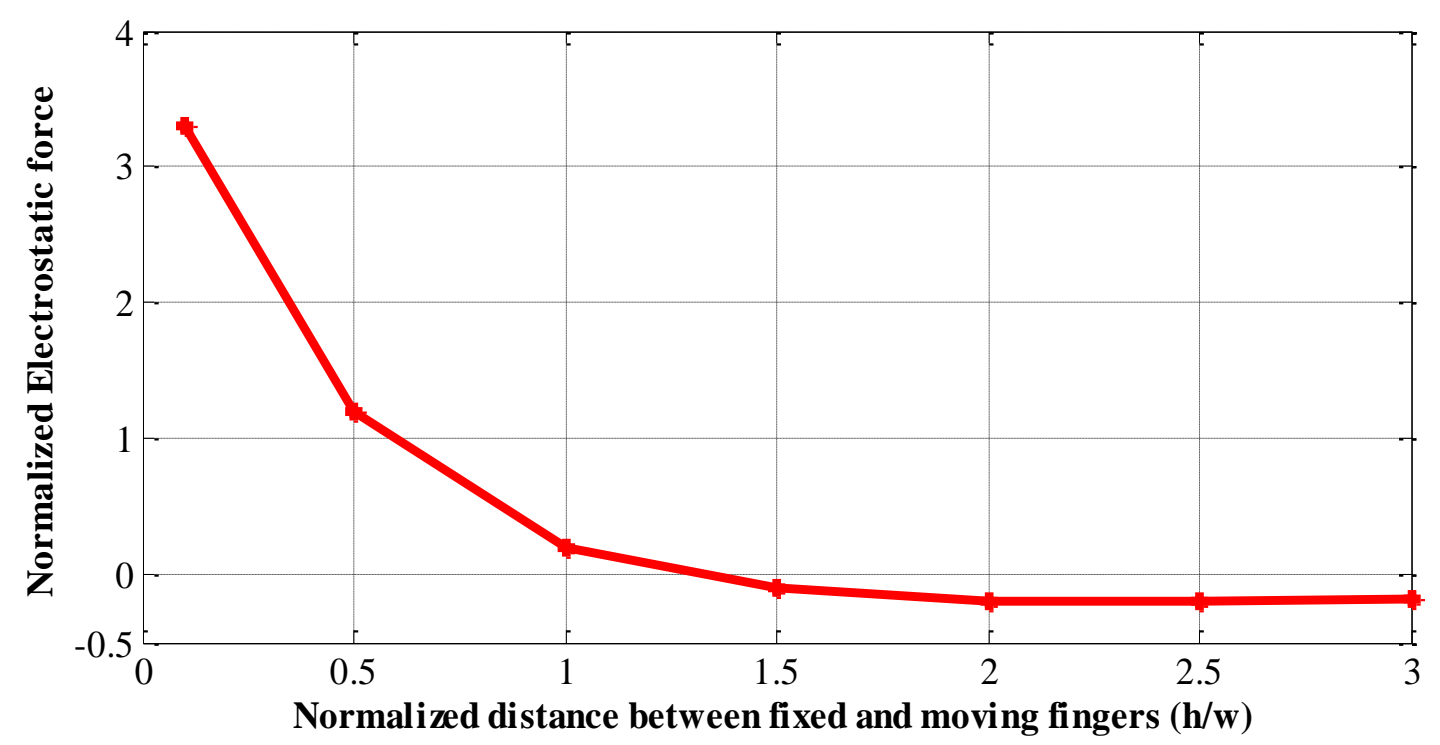

Figure (13): The calculated electrostatic force resulting between fixed and moving fingers.

4.5 The Effect of Lateral Distance Between the Fixed Fingers on the Repulsive Electrostatic Force

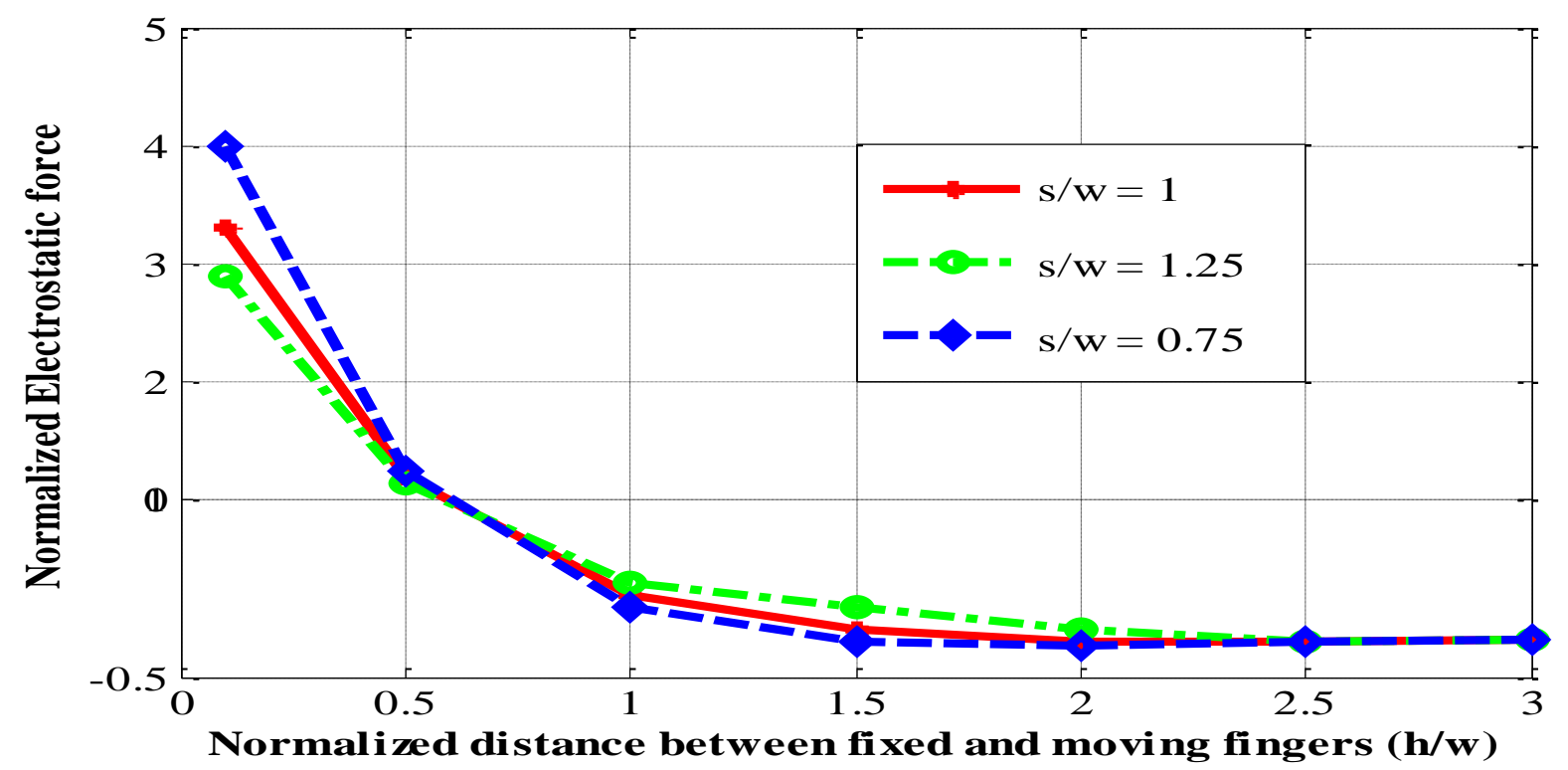

Figure (14): The effect of varying lateral distance on the calculated electrostatic force resulting between fixed and moving fingers.

Decreasing the lateral distance between fixed fingers increases the resulting repulsive force. But decreasing the maximum vertical distance between the fixed finger and the moving finger for generating a repulsive electrostatic force. 


\subsection{The Effect of Plate Thickness of the Moving Fingers}

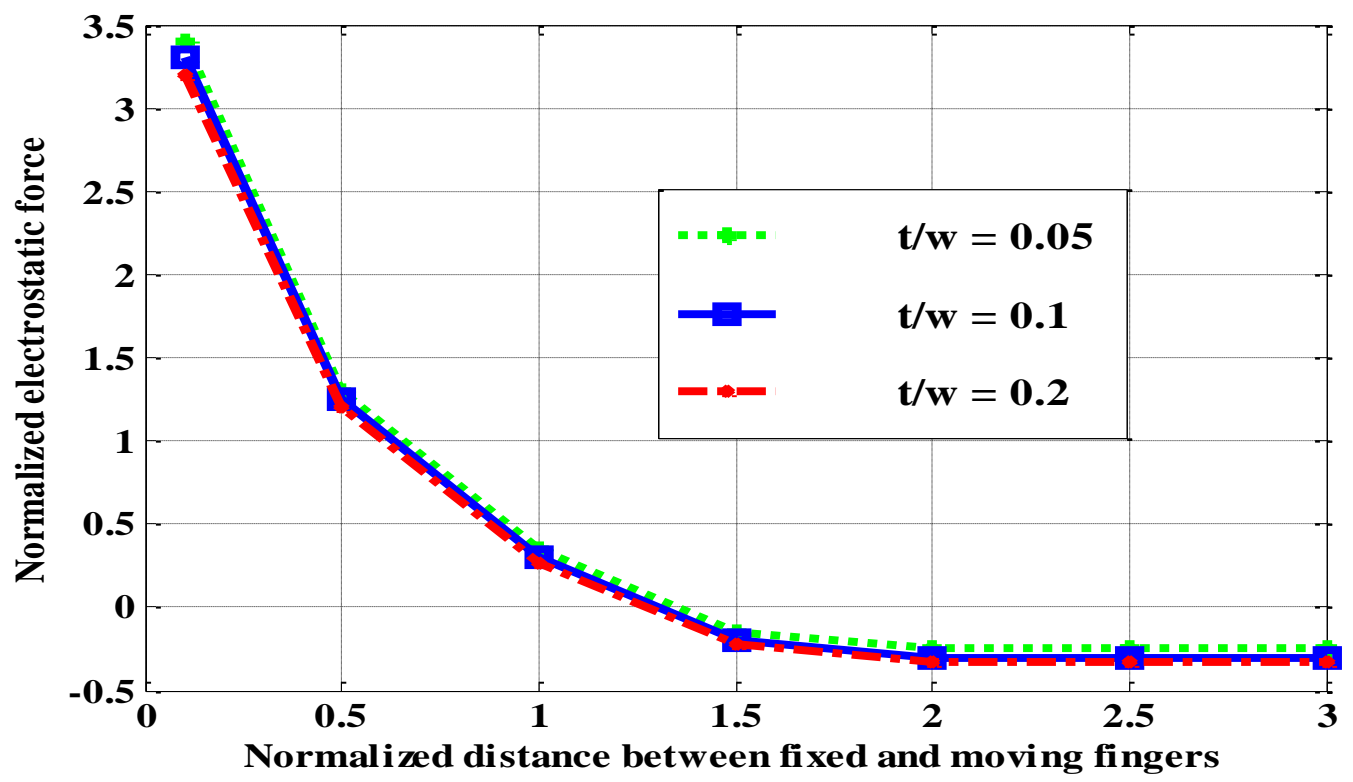

Figure (15): The effect of varying the moving fingers thickness.

Decreasing the thickness of the finger increases the resulting repulsive force produced between the fixed and moving fingers. Also, the maximum vertical distance between the fixed and the moving fingers for generating a repulsive force decreases.

\subsection{The Effect of Applied Voltage on the Repulsive Electrostatic Force.}

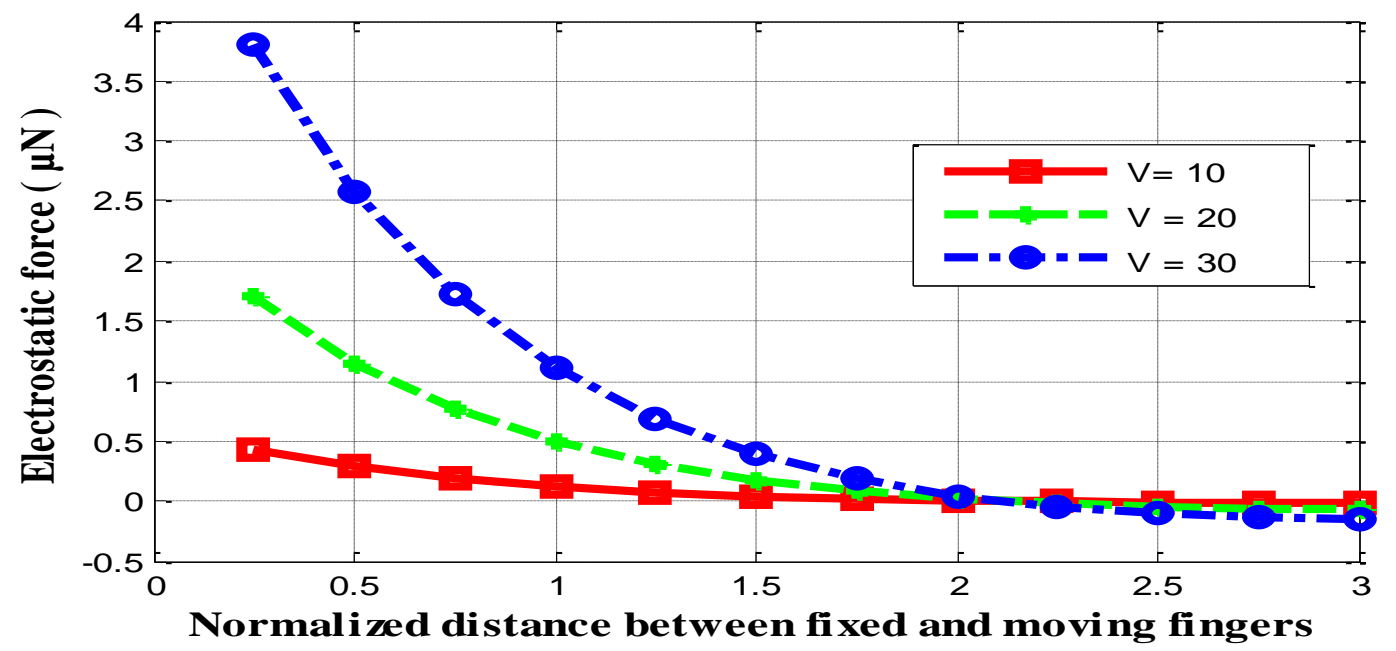

Figure (16): The effect of varying the applied voltage on the calculated electrostatic force resulting between fixed and moving fingers.

Increasing the value of applied voltage, increases the resulting electrostatic force produced between the fixed and the moving fingers. 


\subsection{Discussion of the Results}

According to the results shown in Figures (14-16), the effects of varying the structural parameters $\mathrm{S}, \mathrm{T}$, and $\mathrm{V}$ relative to $\mathrm{w}$ on the microactuator performance are summarized as follows:

a) An increase in the force comes at the cost of a decrease in the maximum displacement of the moving finger or vice versa; and

b) Tuning the microactuator performance through adjusting the scale of the dimension of the cross section of the microactuator is much simpler than varying the structural parameters $\mathrm{S}, \mathrm{T}$, and $\mathrm{V}$, due to the simple mathematical relations between the scale of the dimension of the cross section of the microactuator and the actuator performance

\section{Optimization of Structure Parameters}

Because $\mathrm{H}=\mathrm{h} 0 \mathrm{w}$ is the maximum vertical distance between the fixed and moving fingers for which a repulsive force is generated, the theoretical maximum displacement of the repulsiveforce microactuator can be written as

$$
\mathrm{D}=\mathrm{h}_{0} \mathrm{w}-\mathrm{H}_{\text {initial }}
$$

where $\mathrm{D}$ is the maximum displacement and $\mathrm{H}_{\text {initial }}$ is the initial distance between the fixed and moving fingers when no voltage is applied.

The following rules are derived for tuning and optimizing the performance of the microactuator:

- Rule 1

The maximum displacement of the microactuator is proportional to the dimension of the cross section of the microactuator (w) (26), as well as the lateral distance between fixed fingers. Hence, scaling the dimension of the cross section of the microactuator, i.e., increasing $\mathrm{S}, \mathrm{T}$, and $\mathrm{w}$, proportionally changes the maximum vertical displacement of the microactuator.

- Rule 2

The force generated in the microactuator is inversely proportional to the scale of the dimension of the cross section of the microactuator (w) [(12) and (13)]. In addition, scaling up the dimension of the cross section of the microactuator proportionally reduces the number of moving fingers which can be used for a given microactuator size.

According to Rules 1) and 2), the dimension of the cross section of the microactuator should be determined based on a balance between the desired displacement and required repulsive force.

- Rule 3

The force of the microactuator is proportional to the finger length $(\mathrm{L})$. However, the finger length does not affect the maximum displacement of the microactuator. Therefore, the finger length should be as long as possible in order to achieve a large force within a predefined space and fabrication process limits.

\section{Conclusion}

An analytical model for repulsive-force microactuator has been developed in this paper. The model provides the role of the fringe capacitance, the means to estimate the repulsive force, and reveals the maximum out-of- plane displacement that is achievable by the microactuator. Rules for tuning and optimization of the microactuator performance were developed based on the analytical model. 


\section{References}

[1] Adisorn Tuantranont and Victor M. Bright, "Introduction to Micro-Electro-Mechanical Systems (MEMS) with Emphasis on Optical Applications", NECTEC Technical Journal, Vol. 1, No. 6, pp. 227-236, 2000.

[2] M. Kassem, Mazhar B. Tayel, "New trends in MEMS device applications and marketing", M.S. thesis, Alexandria University, October 2011.

[3] Fangrong Hua,b, Jun Yao a, Chuankai Qiu a, Hao Ren, "A MEMS micromirror driven by electrostatic force", Journal of Electrostatics 68 (2010) 237-242.

[4] S. He, R.B. Mrad, "Large-stroke microelectrostatic actuators for vertical translation of micromirrors used in adaptive optics", J. IEEE Trans. Ind. Electronics 52 (2005) 974-983.

[5] P. W. Cattaneo "Capacitances in micro-strip detectors: A conformal mapping approach" Solid-State Electronics, VOL 54, 2010.

[6] M. F. ElSaadi, "A Proposed model for MEMS tunable capacitor actuators", M.S. thesis, Alexandria University, 2009.

[7] E.Weber, "Electromagnetic Fields, Theory and Applications", New York: Wiley, 1950, vol. I, Mapping of fields.

[8] Aditya Bansal, "An Analytical Fringe Capacitance Model for Interconnects Using Conformal Mapping", IEEE TRANSACTIONS ON COMPUTER-AIDED DESIGN OF INTEGRATED CIRCUITS AND SYSTEMS, VOL. 25, NO. 12, DECEMBER 2006.

[9] R.Plonsey and R. E. Collin, "Principles and Applications of Electromagnetic Fields", New York: McGraw-Hill, 1961. 